\title{
CONTROLE DE FLOR ROXA (Echium plantagineum L.) E SELETIVIDADE À HERBICIDAS PÓS-EMERGENTES EM CULTIVO DE AZEVÉM
}

Rodrigo Roso ${ }^{1 *}$, Nelson Diehl Kruse ${ }^{2}$, Caren Alessandra Müller ${ }^{3}$, Tiele Stuker Fernandes ${ }^{1}$, Eduardo José Ludwig ${ }^{4}$, Pablo Reno da Silva Sangoi ${ }^{3}$, Cassiano Vasconcelos dos Santos ${ }^{3}$

\footnotetext{
${ }^{1}$ Doutorando (a) em Agronomia na Universidade Federal de Santa Maria - Departamento de Fitotecnia - Santa Maria, RS. *Email: rodrigoroso@yahoo.com.br

${ }^{2}$ Professor Doutor da Universidade Federal de Santa Maria - Departamento de Fitotecnia - Santa Maria, RS.

${ }^{3}$ Graduando (a) em Agronomia da Universidade Federal de Santa Maria - Departamento de Fitotecnia - Santa Maria, RS.

${ }^{4}$ Mestrando em Agronomia na Universidade Federal de Santa Maria - Departamento de Fitotecnia - Santa Maria, RS.
}

RESUMO: O azevém (Lolium multiflorum Lam.) é uma gramínea de clima subtropicaltemperado que apresenta bom desenvolvimento em qualquer tipo de solo, sendo muito utilizado como pastagem visando suprir o vazio forrageiro durante o período de inverno no RS. No entanto, algumas plantas daninhas interferem no desenvolvimento dessa cultura, como por exemplo, Echium plantagineum, que é uma espécie herbácea, bianual, pertencente à família Boraginaceae. O presente trabalho teve como objetivo avaliar a eficácia de herbicidas pós-emergentes, no controle de E. plantagineum e a fitotoxicidade na cultura do azevém. O experimento foi desenvolvido a campo durante os meses de julho e agosto de 2014. No local do experimento foi realizado o cultivo de azevém e nessa área ocorria alta infestação da planta daninha E. plantagineum. Os tratamentos avaliados foram: saflufenacil (28 e $56 \mathrm{~g} \mathrm{ha}^{-1}$ de i.a); 2,4-D (806 e $1612 \mathrm{~g} \mathrm{ha}^{-1}$ de i.a); metsulfurom-metílico (3 e $6 \mathrm{~g} \mathrm{ha}^{-1}$ de i.a); bentazona (600 e $900 \mathrm{~g} \mathrm{ha}^{-1}$ de i.a) e 2,4-D + metsulfurom-metílico (806 + $3 \mathrm{~g} \mathrm{ha}^{-1}$ de i.a). Os tratamentos foram aplicados em pós-emergência do azevém e de $E$. plantagineum, com pulverizador costal pressurizado por $\mathrm{CO}_{2}$, equipado com barra de quatro pontas espaçadas $0,50 \mathrm{~m}$, calibrado para a taxa de aplicação de $150 \mathrm{~L} \mathrm{ha}^{-1}$. As variáveis analisadas foram controle de E. plantagineum, fitotoxicidade no azevém e massa seca da parte aérea (MSPA) de ambas as espécies. O herbicida 2,4-D apresentou os melhores percentuais de controle de E. plantagineum em ambas as doses utilizadas, não proporcionando fitotoxicidade na cultura do azevém. Já o herbicida saflufenacil apresentou controle ineficiente de E. plantagineum e proporcionou fitotoxicidade moderada no azevém, acarretando em diminuição da MSPA quando comparado aos tratamentos com 2,4-D.

Palavras-chave: Boraginaceae. Planta daninha. Poaceae. Pós-emergentes.

\section{CONTROL OF PURPLE FLOWER (Echium plantagineum L.) AND SELECTIVITY TO POST-EMERGING HERBICIDES IN CULTIVATION OF ITALIAN RYEGRASS}

Cultura Agronômica, Ilha Solteira, v.26, n.3, p.297-309, 2017 


\begin{abstract}
Italian ryegrass (annual ryegrass) (Lolium multiflorum Lam.) is a grass from temperate subtropical climate that presents good development in any type of soil, commonly used as pasture to fill the forage void during the winter in the RS. However, some weeds interfere in the development of this plant, for example, Echium plantagineum, which is a biennial herbaceous species belonging to the family Boraginaceae. Therefore, this study aimed to evaluate the efficacy of post-emerging herbicides to control E. plantagineum and phytotoxicity in cultivation of italian ryegrass. To do this, we performed a field experiment during the months of July and August 2014. In the local of the experiment, with high infestation of the weed E. plantagineum, Italian ryegrass was cultivated. The evaluated treatments were: saflufenacil (28 and $56 \mathrm{~g} \mathrm{ha}^{-1}$ of a.i); 2,4-D (806 and $1612 \mathrm{~g} \mathrm{ha}^{-1}$ of a.i); metsulfurom-methyl (3 and $6 \mathrm{~g} \mathrm{ha}^{-1}$ of a.i); bentazone (600 and $900 \mathrm{~g} \mathrm{ha}^{-1}$ of a.i) and 2,4- D + metsulfurom-methyl $\left(806+3 \mathrm{~g} \mathrm{ha}^{-1}\right.$ of a.i). The treatments were applied post-emerging of italian ryegrass and E. plantagineum, with $\mathrm{CO}_{2}$ pressurized costal sprayer, equipped with 0,50 m spaced four-point bar, calibrated for the application rate of $150 \mathrm{~L} \mathrm{ha}^{-1}$. The analyzed variables were the control of E. plantagineum, phytotoxicity on italian ryegrass and shoot dry matter (SDM) of both species. The herbicide 2,4-D presented the highest percentage of control of E. plantagineum in both used doses, resulting in no phytotoxicity on cultivation of italian ryegrass. In the other hand, saflufenacil herbicide was inefficient to control $E$. plantagineum and presented moderate phytotoxicity in italian ryegrass, causing a decrease SDM compared to treatment with 2,4-D.
\end{abstract}

Key words: Boraginaceae. Weed. Poaceae. Post-emerging.

\title{
INTRODUÇÃO
}

O azevém anual (Lolium multiflorum Lam.) tem grande importância nos sistemas produtivos pecuários do sul do Brasil e constitui-se na espécie forrageira de estação fria mais utilizada no Rio Grande do Sul (RS) (VARGAS et al., 2007; CONFORTIN et al., 2013). A utilização de azevém permite a criação de animais em regime de pastagem nesse período crítico, e consiste no método mais barato de fornecer alimentação abundante e de boa qualidade nutricional (ROMAN et al., 2007). É uma espécie rústica e vigorosa, perfilha em abundância, sendo uma importante alternativa para amenizar o vazio forrageiro durante o outono-inverno no sul do país (FERRAZZA et al., 2013).

Além de ser uma abundante fonte de forragem, o azevém possui elevada tolerância ao pisoteio e ao pastejo de até cinco meses, possuindo também tolerância a doenças e sistema radicular vigoroso (BOLDRINI et al., 2008). Possui boa capacidade de rebrote, ressemeadura natural e apresenta elevado valor nutritivo, podendo ser utilizado para compor pastagens com diversas outras espécies (DE CONTO et al., 2011). Também apresenta alta qualidade da forragem, sendo que a proteína bruta na matéria seca varia de 17 a $24 \%$, e a digestibilidade da matéria orgânica varia de 69 a 81\% (FARINATTI et al., 2006).

Cultura Agronômica, Ilha Solteira, v.26, n.3, p.297-309, 2017 
Em pastagens cultivadas, alguns fatores contribuem para a baixa produtividade e qualidade da forragem, dentre os quais, à presença de plantas daninhas interferem no crescimento e desenvolvimento das pastagens (SANTOS et al., 2015). Como por exemplo, E. plantagineum (flor roxa), que é uma espécie herbácea, bianual, pertencente à família Boraginaceae (SAINT-HILAIRE, 2009). A espécie é desprovida de caule, apresentando no início do ciclo apenas uma grande roseta de folhas, sendo as folhas inferiores sésseis e as superiores apresentam curto pecíolo, ambas com formato lanceolado (MOREIRA; BRAGANÇA, 2010). Quando em alta infestação formam densas monoculturas, capazes de produzir grandes quantidades de sementes que podem germinar a qualquer momento do ano, e permanecer por até seis anos dormentes no solo (WESTON et al., 2012). Essa planta daninha além de competir com as plantas cultivadas por nutrientes, espaço e luz, contém alcalóides pirazólicos em suas folhas tornando-a potencialmente tóxica para alguns herbívoros, como cavalos e ovinos, podendo causar encefalopatia hepática (SANTOS et al., 2008).

Para o controle de plantas dicotiledôneas em pastagens cultivadas, como de azevém por exemplo, não existe recomendação específica, sendo raras as informações de seletividade de herbicidas para o azevém como cultura (DALAZEN et al., 2015). Pois, na maioria das vezes o azevém é considerado planta daninha das culturas de cereais de inverno. Baseado nisso, objetivou-se com este trabalho avaliar a eficiência de herbicidas, no controle de E. Plantagineum e na seletividade da pastagem cultivada de azevém, aplicados em pósemergência do azevém e da planta daninha.

\section{MATERIAL E MÉTODOS}

O experimento foi desenvolvido a campo nos meses de julho e agosto do ano de 2014. O mesmo foi realizado no município de Restinga Seca, RS, situado a $72 \mathrm{~m}$ de altitude, $29^{\circ}$ 51' 29" S e 53 31' 41" O. O solo da área experimental foi classificado como Argissolo Vermelho distrófico típico conforme normas da Embrapa (2006), com relevo suave ondulado. O clima é caracterizado, segundo a classificação de Köppen, como subtropical úmido (Cfa), sem estação seca, com temperatura média do mês mais quente superior a $22^{\circ} \mathrm{C}$ (MORENO, 1961). As precipitações são regulares ao longo do ano, com índices pluviométricos médios anuais de $1700 \mathrm{~mm}$.

A área experimental foi cultivada com pastagem de azevém comum provindo de ressemeadura natural, sem ocorrência de plantas de azevém resistentes, possuindo soja como cultura antecessora. A área apresenta histórico de infestação por E. plantagineum por pelo menos 10 anos, com população média de $20 \mathrm{pl} \mathrm{m}^{-2}$. Os tratamentos foram aplicados dia 08 de julho de 2014, nesta data as plantas de E. plantagineum estavam no estádio de roseta, possuindo diâmetro médio de $15 \mathrm{~cm}$, e o azevém estavam em pleno perfilhamento. 
Foi realizada adubação de cobertura, no início do perfilhamento do azevém, de $100 \mathrm{~kg}$ $\mathrm{ha}^{-1}$ do fertilizante 2-25-25 (NPK), mais uma aplicação de $50 \mathrm{~kg} \mathrm{ha}^{-1}$ de uréia com $45 \%$ de nitrogênio. Os tratamentos aplicados estão descritos na Tabela 1.

Tabela 1. Ingrediente ativo, concentração e dose dos herbicidas aplicados para o controle de Echium plantagineum em pastagem cultivada de azevém.

\begin{tabular}{|c|c|c|c|c|}
\hline $\mathrm{N}^{\circ}$ & Ingrediente ativo & Concentração & $\begin{array}{c}\text { Dose } \mathrm{g} \mathrm{ha}^{-1} \\
\text { (i.a.) }\end{array}$ & $\begin{array}{c}\text { Dose de p.c. } \\
\text { ha }^{-1}\end{array}$ \\
\hline 1 & Testemunha & - & - & - \\
\hline 2 & Saflufenacil $^{1}$ & $700 \mathrm{~g} \mathrm{~kg}^{-1}$ & 28 & $40 \mathrm{~g}$ \\
\hline 3 & Saflufenacil & $700 \mathrm{~g} \mathrm{~kg}^{-1}$ & 56 & $80 \mathrm{~g}$ \\
\hline 4 & 2,4-D & $806 \mathrm{~g} \mathrm{~L}^{-1}$ & 806 & $1 \mathrm{~L}$ \\
\hline 5 & $2,4-\mathrm{D}$ & $806 \mathrm{~g} \mathrm{~L}^{-1}$ & 1612 & $2 \mathrm{~L}$ \\
\hline 6 & Metsulfurom-metílico ${ }^{2}$ & $600 \mathrm{~g} \mathrm{~kg}^{-1}$ & 3 & $5 \mathrm{~g}$ \\
\hline 7 & Metsulfurom-metílico & $600 \mathrm{~g} \mathrm{~kg}^{-1}$ & 6 & $10 \mathrm{~g}$ \\
\hline 8 & Bentazona $^{3}$ & $600 \mathrm{~g} \mathrm{~L}^{-1}$ & 600 & $1 \mathrm{~L}$ \\
\hline 9 & Bentazona & $600 \mathrm{~g} \mathrm{~L}^{-1}$ & 900 & $1,5 \mathrm{~L}$ \\
\hline 10 & 2,4-D + Metsulfurom-metílico & $806 \mathrm{~g} \mathrm{~L}^{-1}+600 \mathrm{~g} \mathrm{~kg}^{-1}$ & $806+3$ & $1 \mathrm{~L}+5 \mathrm{~g}$ \\
\hline
\end{tabular}

${ }^{1}$ adição de Dash $^{\circledR}$ adjuvante não iônico a $0,5 \%$ v/v; ${ }^{2}$ adição de óleo mineral emulsionável à calda de pulverização a $0,1 \% \mathrm{v} / \mathrm{v} ;{ }^{3}$ adição do adjuvante oleoso Assist ${ }^{\circledR}$ na dose de $1,0 \mathrm{~L} \mathrm{ha}^{-1}$.

O delineamento experimental adotado foi de blocos ao acaso com dez tratamentos e quatro repetições, possuindo cada unidade experimental dimensões de 2,5 metros de largura por cinco metros de comprimento, totalizando $12,5 \mathrm{~m}^{2}$. Os tratamentos foram aplicados em pós-emergência da cultura do azevém e das plantas de E. plantagineum, com pulverizador costal pressurizado por $\mathrm{CO}_{2}$, equipado com barra de quatro pontas espaçadas $0,50 \mathrm{~m}$, possuindo pontas do tipo leque jato plano XR TeeJet -110.02 , calibrado para a taxa de aplicação de $150 \mathrm{~L} \mathrm{ha}^{-1}$. Durante a aplicação dos tratamentos a umidade relativa do ar permaneceu $68 \pm 5 \%$ e temperatura de $20 \pm 2^{\circ} \mathrm{C}$. No momento das aplicações o céu estava aberto sem a presença de nuvens e a velocidade do vento se manteve entre 0 e $6 \mathrm{~km} \mathrm{~h}^{-1}$.

Os parâmetros avaliados foram, controle percentual de E. plantagineum aos 7, 14, 21 e 28 dias após a aplicação dos tratamentos (DAT). O controle foi avaliado utilizando escala visual percentual, variando de zero (0) a cem (100\%), onde zero corresponde à ausência total de sintomas e cem $(100 \%)$ ao controle total de E. plantagineum na área (FRANS, 1986). Também foi avaliado, a seletividade dos herbicidas na cultura do azevém em relação aos sintomas de amarelecimento, necrose das folhas e vigor das plantas, através da fitotoxicidade, aos 7, 14, 21 e 28 DAT dos herbicidas, sendo determinada por comparação visual, em que foi atribuída a nota de zero (ausência total de fitotoxicidade) a cem (100\%) (morte das plantas). Além disso, foi avaliada a massa seca da parte aérea (MSPA), sendo realizada uma coleta de matéria verde em uma área de $0,25 \mathrm{~m}^{2}$ alocadas aleatoriamente em cada unidade experimental. Nesta área, foi coletada separadamente a parte aérea das plantas de azevém e E. plantagineum, e posteriormente secas em estufa com circulação de ar 
forçada a $65{ }^{\circ} \mathrm{C}$ por 5 dias para a determinação da MSPA de ambas espécies. Após secas, as amostras foram pesadas em uma balança de precisão de dois dígitos.

Os dados foram submetidos à aplicação do teste $\mathrm{F}$ na análise da variância, seguido pelo teste de Tukey, com 5\% de probabilidade. Os dados das variáveis de fitotoxicidade das plantas de azevém e controle de E. plantagineum aos 7, 14, 21 e 28 DAT foram transformados em $\operatorname{arcsen} \sqrt{ }(\mathrm{x}+\mathrm{k})$, a fim de se corrigir a heterocedasticidade.

\section{RESULTADOS E DISCUSSÃO}

A testemunha (sem aplicação) foi parâmetro de referência para as avaliações visuais de controle de E. plantagineum e de fitotoxicidade das plantas de azevém nas avaliações realizadas aos 7, 14, 21 e 28 DAT.

Tabela 2. Avaliação visual de controle (\%) de Echium plantagineum em pastagem de azevém, aos 7, 14, 21 e 28 DAT (dias após a aplicação dos tratamentos), em resposta à aplicação de diferentes herbicidas e doses.

\begin{tabular}{|c|c|c|c|c|c|c|}
\hline \multirow[t]{2}{*}{$\mathrm{N}^{\circ}$} & \multirow{2}{*}{ Tratamentos } & \multirow{2}{*}{ Dose $\mathrm{g} \mathrm{ha}^{-1}$ (i.a.) } & \multicolumn{4}{|c|}{ Controle $(\%)^{* *}$} \\
\hline & & & $7 \mathrm{DAT}$ & $14 \mathrm{DAT}$ & $21 \mathrm{DAT}$ & $28 \mathrm{DAT}$ \\
\hline 1 & Testemunha & - & $0,0 \mathrm{e}^{*}$ & $0,0 \mathrm{f}$ & $0,0 \mathrm{c}$ & $0,0 \mathrm{~d}$ \\
\hline 2 & Saflufenacil & 28 & 40,0 a & $52,5 \mathrm{~b}$ & 68,8 a & $71,3 \mathrm{~b}$ \\
\hline 3 & Saflufenacil & 56 & 38,8 a & 60,0 a & 70,0 a & $71,3 \mathrm{~b}$ \\
\hline 4 & 2,4-D & 806 & $22,5 \mathrm{bc}$ & $42,5 \mathrm{c}$ & 66,3 a & 86,3 a \\
\hline 5 & $2,4-\mathrm{D}$ & 1612 & $28,8 \mathrm{~b}$ & $51,3 \mathrm{~b}$ & 73,8 a & 90,0 a \\
\hline 6 & Metsulfurom-metílico & 3 & $10,0 \mathrm{~d}$ & $28,8 \mathrm{e}$ & $45,0 \mathrm{~b}$ & $66,3 \mathrm{bc}$ \\
\hline 7 & Metsulfurom-metílico & 6 & $10,0 \mathrm{~d}$ & $28,8 \mathrm{e}$ & $45,0 \mathrm{~b}$ & $72,5 \mathrm{~b}$ \\
\hline 8 & Bentazona & 600 & $17,5 \mathrm{c}$ & 31,3 de & $47,5 \mathrm{~b}$ & $57,5 \mathrm{c}$ \\
\hline 9 & Bentazona & 900 & $16,3 \mathrm{c}$ & $37,5 \mathrm{~cd}$ & $48,8 \mathrm{~b}$ & 58,8 c \\
\hline 10 & 2,4-D + Metsulfurom-metílico & $806+3$ & $18,8 \mathrm{c}$ & $42,5 \mathrm{c}$ & 67,5 a & 88,8 a \\
\hline & $\mathrm{CV}(\%)^{* * *}$ & & 7,32 & 4,28 & 4,50 & 5,25 \\
\hline
\end{tabular}

* Médias seguidas pela mesma letra, na coluna, não diferem significativamente pelo teste de Tukey ao nível de $5 \%$ de probabilidade. ${ }^{* *}$ Percentuais de controle transformados por $\operatorname{arcsen} \sqrt{ }(\mathrm{x}+\mathrm{k}) .{ }^{* * *}$ Coeficiente de variação percentual.

Nas avaliações de controle de E. plantagineum em pastagem cultivada de azevém, que iniciaram aos 7 DAT, pode-se observar que o herbicida saflufenacil (28 e $56 \mathrm{~g} \mathrm{ha}^{-1}$ de i.a) apresentou o melhor controle dessa planta daninha em relação aos demais herbicidas aos 7 DAT, atingindo percentuais de controle de 40\% (Tabela 2). Essa ação rápida do saflufenacil é característica de herbicidas inibidores da enzima protoporfirinogênio oxidase (PPO), podendo apresentar necroses visíveis nas folhas 48 horas após a aplicação do herbicida (GROSSMANN et al., 2011). Já nas avaliações aos 14, 21 e 28 DAT, pode-se verificar que esse herbicida passou a ter controle semelhante ou inferior aos tratamentos com o herbicida 2,4-D (Tabela 2). Dessa forma, aos 14 DAT nas doses de 28 e $56 \mathrm{~g} \mathrm{ha}^{-1}$ de i.a. a eficiência

Cultura Agronômica, Ilha Solteira, v.26, n.3, p.297-309, 2017 
atingiu 52,5 e 60,0\% sobre o controle das plantas de E. plantagineum, respectivamente. Aos 21 DAT a eficiência no controle atingiu 68,8 e 70,0\% para doses de 28 e $56 \mathrm{~g} \mathrm{ha}^{-1}$ de i.a. respectivamente, e aos 28 DAT alcançou percentuais de $71,3 \%$ para ambas as doses do herbicida (Tabela 2), sendo considerado o controle moderado das plantas de $E$. plantagineum.

No entanto, aos 21 DAT observou-se o início da rebrota de algumas plantas de $E$. plantagineum, comprovando sua capacidade de recuperação e ineficácia do herbicida saflufenacil no controle, quando as plantas possuíram diâmetro igual ou superior a $20 \mathrm{~cm}$ no estádio de roseta. Resultados semelhantes foram encontrados por Vitorino et al. (2012) em trabalho realizado com jato dirigido na cultura da mamona, onde as plantas de Richardia brasilienses e Sida rhombifolia apresentaram rebrota aos 21 DAT, ocorrendo queda no controle dos tratamentos com saflufenacil nas doses de 28,40 e $56 \mathrm{~g} \mathrm{ha}^{-1}$ de i.a. Piccinini et al. (2012) em trabalho com aplicações isoladas ou associadas de glifosato e saflufenacil, observaram sintomas de rebrote das plantas de Ipomoea triloba a partir dos 15 DAT nas menores doses de saflufenacil (2,8 e 5,6 $\mathrm{g} \mathrm{ha}^{-1}$ de i.a.), quando aplicado isolado, diminuindo o controle em relação as avaliações anteriores. Além desses, Inoue et al. (2012) também observou rebrota acentuada das plantas de Crotalaria spectabilis aos 21 DAT, atingindo percentuais de $47,5 \%$ de rebrota aos 42 DAT, quando trabalhou com a mistura de glifosato e saflufenacil (1240 e 52,5 $\mathrm{g} \mathrm{ha}^{-1}$ de i.a.). Esses resultados indicam que o saflufenacil apresenta ação rápida na planta, no entanto, por ter baixa atividade sistêmica pode possuir ineficácia de controle de plantas daninhas em estádios mais avançados, não sendo eficiente se as mesmas apresentarem reservas nas estruturas, podendo facilitar a rebrota.

Os tratamentos com o herbicida 2,4-D aos 7 DAT apresentaram controle pobre, no entanto foram inferiores apenas aos tratamentos com saflufenacil, sendo que para as doses de 806 e $1612 \mathrm{~g} \mathrm{ha}^{-1}$ de i.a. os percentuais de controle foram de 22,5 e 28,5\% respectivamente (Tabela 2). Contudo, nas avaliações aos 21 DAT os tratamentos com 2,4-D apresentaram controle moderado a satisfatório, obtendo valores de $73,8 \%$ para a maior dose (1612 $\mathrm{g} \mathrm{ha}^{-1}$ de i.a.). Para a avaliação aos 28 DAT os tratamentos com 2,4-D possuíram controle de satisfatório a excelente com percentuais de 86,3 e 90,0\% para a menor e a maior dose (806 e $1612 \mathrm{~g} \mathrm{ha}^{-1}$ de i.a.) respectivamente (Tabela 2). Sendo esses, os tratamentos que possuíram melhores controles de E. plantagineum, juntamente com a combinação do 2,4-D + metsulfurom-metílico que apresentou controle satisfatório (88,8\%) aos 28 DAT.

Esses dados mostram que tanto as aplicações de 2,4-D isoladas ou em mistura com metsulfurom-metílico, são eficientes no controle de E. plantagineum possuindo controles considerados satisfatório a excelente. Estes resultados corroboram com os resultados obtidos por Farinelli et al. (2005), que obteve aos 14 DAT resultados de 87,2\% de controle de Raphanus raphanistrum na cultura do milheto quando aplicou $670 \mathrm{~g} \mathrm{ha}^{-1}$ de i.a. de 2,4-D. Costa e Rizzardi (2013) também encontraram resultados similares de controle de Raphanus raphanistrum, onde aos 14 DAT conseguiram 95,0\% de controle dos biótipos suscetíveis a herbicidas inibidores de ALS. Além destes, Takano et al. (2013) verificou que no controle 
de Commelina benghalensis com o herbicida 2,4-D a porcentagem de controle aos 28 DAT foi de $97,5 \%$ com as plantas de $C$. benghalensis possuindo de duas a quatro folhas, já com quatro a seis folhas o controle diminuiu para $74,2 \%$ e acima de dez folhas o controle foi de apenas $27,5 \%$, isso mostra a dificuldade de controle das plantas com estádio avançado de desenvolvimento. $\mathrm{O}$ mesmo pode ter ocorrido neste trabalho, pois devido à ocorrência de algumas plantas de E. plantagineum em estádio avançado de desenvolvimento $(\geq 20 \mathrm{~cm}$ de diâmetro) o controle não ultrapassou 90,0\% no tratamento mais eficiente (1612 $\mathrm{g} \mathrm{ha}^{-1} \mathrm{de}$ i.a. de 2,4-D).

As avaliações nos tratamentos com metsulfurom-metílico demonstraram que o nível de controle de E. plantagineum iniciou-se aos 7 DAT com eficiência de 10,0\% para ambas as doses (3 e $6 \mathrm{~g} \mathrm{ha}^{-1}$ de i.a.) (Tabela 2). Aos 14 DAT o controle atingiu 28,8\% e aos 21 DAT evoluiu para 45,0\% para ambas as doses do herbicida. Atingindo controle moderado aos 28 DAT com percentuais de 66,3 e 75,5\% controle para as doses de 3 e $6 \mathrm{~g} \mathrm{ha}^{-1}$ de i.a., respectivamente. Os baixos percentuais de controle de E. plantagineum pelo herbicida metsulfurom-metílico pode estar atrelado a tolerância dessas plantas daninhas a este herbicida e ao estádio avançado em que se encontravam parte das plantas de $E$. plantagineum, demonstrando a dificuldade do controle desta espécie.

Para os tratamentos com a aplicação de bentazona os percentuais de controle de $E$. plantagineum aos 7 DAT iniciaram com 17,5 e 16,5\% para a menor e maior dose do herbicida (600 e $900 \mathrm{~g} \mathrm{ha}^{-1}$ de i.a.). Sendo que, aos 28 DAT o controle não ultrapassou $58,8 \%$ para a maior dose do herbicida (Tabela 2). Esses resultados mostram que os tratamentos com aplicação de bentazona possuíram menor eficiência no controle de $E$. plantagineum em pastagem cultivada de azevém, não sendo eficiente no controle dessa planta daninha. Resultados similares foram encontrados por Monquero e Christoffolet (2001) em trabalho conduzido em casa de vegetação, onde encontraram percentuais de controle de 58,0\% para plantas de Amaranthus quitensis quando aplicaram $480 \mathrm{~g} \mathrm{ha}^{-1}$ de i.a. de bentazona. Segundo esses mesmos autores, em trabalho conduzido por eles a campo, avaliando o controle de picão-preto e obtiveram controle de $72,0 \%$ dessa planta daninhas quando aplicado $480 \mathrm{~g} \mathrm{ha}^{-1}$ de i.a. de bentazona.

Em relação às avaliações visuais de fitotoxicidade das plantas de azevém, pode-se verificar que aos 7 DAT (Tabela 3) o herbicida saflufenacil apresentou a maior fitointoxicação para a cultura do azevém, com percentuais de 36,3 e 35,0\% para as doses de 28 e $56 \mathrm{~g} \mathrm{ha}^{-1}$ de i.a., respectivamente, sendo observado clorose moderada nas folhas de azevém e leve redução na estatura das plantas. Já os tratamentos com 2,4-D, 2,4-D + metsulfurom-metílico e bentazona (900 $\mathrm{g} \mathrm{ha}^{-1}$ de i.a.) apresentaram fitotoxicidade leve (Tabela 3), apenas apresentando clorose nas folhas, porém de fraca intensidade. Nos demais tratamentos não foram observados sintomas de fitotoxicidade na cultura.

Aos 14 DAT, observou-se de forma geral que os efeitos de fitotoxicidade continuaram na maioria dos tratamentos sendo, que para os tratamentos com saflufenacil os sintomas de clorose permaneceram e observou-se também, nítida redução de estatura das plantas de 
azevém. Além disso, os tratamentos com metsulfurom-metílico passaram a apresentar fitointoxicação nas plantas de azevém, através de leve clorose e redução da estatura das plantas, com percentuais de 20,0\% para ambas as doses ( 3 e $6 \mathrm{~g} \mathrm{ha}^{-1}$ de i.a.). Além destes, o tratamento com bentazona na dose de $600 \mathrm{~g} \mathrm{ha}^{-1}$ de i.a. também passou a evidenciar sintomas de fitointoxicação leve (Tabela 3), semelhante ao outro tratamento com bentazona (900 $\mathrm{g} \mathrm{ha}^{-1}$ de i.a.).

Tabela 3. Avaliação visual de fitotoxicidade (\%) nas plantas de azevém, aos 7, 14, 21 e 28 DAT (dias após a aplicação dos tratamentos), em resposta à aplicação de diferentes herbicidas e doses.

\begin{tabular}{|c|c|c|c|c|c|c|}
\hline \multirow{2}{*}{$\mathrm{N}^{\circ}$} & \multirow{2}{*}{ Tratamentos } & \multirow{2}{*}{ Dose $\mathrm{g} \mathrm{ha}^{-1}$ (i.a.) } & \multicolumn{4}{|c|}{ Fitotoxicidade $(\%)^{* *}$} \\
\hline & & & 7 DAT & $14 \mathrm{DAT}$ & $21 \mathrm{DAT}$ & $28 \mathrm{DAT}$ \\
\hline 1 & Testemunha & - & $0,0 \mathrm{c}^{*}$ & $0,0 \mathrm{~d}$ & $0,0 \mathrm{~b}$ & $0,0 \mathrm{c}$ \\
\hline 2 & Saflufenacil & 28 & $36,3 \mathrm{a}$ & $36,3 \mathrm{a}$ & $28,8 \mathrm{a}$ & $21,3 \mathrm{a}$ \\
\hline 3 & Saflufenacil & 56 & $35,0 \mathrm{a}$ & $37,5 \mathrm{a}$ & $31,3 \mathrm{a}$ & $18,8 \mathrm{ab}$ \\
\hline 4 & 2,4-D & 806 & $11,3 \mathrm{~b}$ & $10,0 \mathrm{c}$ & $0,0 \mathrm{~b}$ & $0,0 \mathrm{c}$ \\
\hline 5 & $2,4-\mathrm{D}$ & 1612 & $13,8 \mathrm{~b}$ & $10,0 \mathrm{c}$ & $0,0 \mathrm{~b}$ & $0,0 \mathrm{c}$ \\
\hline 6 & Metsulfurom-metílico & 3 & $0,0 \mathrm{c}$ & $20,0 \mathrm{~b}$ & $27,5 \mathrm{a}$ & $20,0 \mathrm{ab}$ \\
\hline 7 & Metsulfurom-metílico & 6 & $0,0 \mathrm{c}$ & $20,0 \mathrm{~b}$ & $27,5 \mathrm{a}$ & $21,3 \mathrm{a}$ \\
\hline 8 & Bentazona & 600 & $0,0 \mathrm{c}$ & $12,5 \mathrm{c}$ & $0,0 \mathrm{~b}$ & $0,0 \mathrm{c}$ \\
\hline 9 & Bentazona & 900 & $11,3 \mathrm{~b}$ & $11,3 \mathrm{c}$ & $0,0 \mathrm{~b}$ & $0,0 \mathrm{c}$ \\
\hline 10 & 2,4-D + Metsulfurom-metílico & $806+3$ & $13,8 \mathrm{~b}$ & $13,8 \mathrm{c}$ & $27,5 \mathrm{a}$ & $15,0 \mathrm{~b}$ \\
\hline & $\mathrm{CV}(\%) * * *$ & & 7,07 & 6,13 & 6,83 & 10,87 \\
\hline
\end{tabular}

* Médias seguidas pela mesma letra, na coluna, não diferem significativamente pelo teste de Tukey ao nível de $5 \%$ de probabilidade. $* *$ Percentuais de controle transformados por $\operatorname{arcsen} \sqrt{ }(\mathrm{x}+\mathrm{k}) .{ }^{* * *}$ Coeficiente de variação percentual.

$\mathrm{Na}$ avaliação realizada aos 21 DAT, observou-se que os tratamentos com saflufenacil apresentaram leve redução de fitointoxicação das plantas de azevém, passando a possuir percentuais de 28,8 e $31,3 \%$ de fitotoxicidade para a dose de 28 e $56 \mathrm{~g} \mathrm{ha}^{-1}$ de i.a., respectivamente. Cabe ressaltar também que os tratamentos com metsulfurom-metílico e 2,4-D + metsulfurom-metílico passaram a apresentar sintomas mais pronunciados de fitotoxicidade, com percentuais de 27,5\% para ambos os tratamentos (Tabela 3). Já para 2,4D e bentazona os sintomas de fitotoxicidade foram superados pela cultura do azevém.

Aos 28 DAT, de forma geral todos os tratamentos possuíram redução da fitotoxicidade. Observou-se que os tratamentos com saflufenacil e metsulfurom-metílico apresentaram percentuais semelhantes de fitotoxicidade ao redor de $20,0 \%$, possuindo em ambos os tratamentos sintomas leves de clorose e redução de estatura das plantas (Tabela 3). Sintomas semelhantes foram encontrados no tratamento com 2,4-D + metsulfurom-metílico $\left(806+3 \mathrm{~g} \mathrm{ha}^{-1}\right.$ de i.a.), apresentando este 15,0\% de fitotoxicidade, provavelmente em decorrência do herbicida metsulfurom-metílico. Os demais tratamentos neste período não possuíram sintomas de fitotoxicidade. 
Embora este trabalho tenha apresentado fitotoxicidade mais pronunciada de saflufenacil na cultura do azevém, em trabalhos realizados por Dalazen et al. (2015) com a aplicação de 35 e $70 \mathrm{~g} \mathrm{ha}^{-1}$ de i.a. de saflufenacil, o autor encontrou aos 10 DAT respectivamente apenas 5,0 e 10,0\% de fitotoxicidade nas plantas de azevém comum. Esse mesmo autor também não encontrou fitotoxicidade do metsulfurom-metílico nas plantas de azevém nas doses de 2,4 e 4,8 $\mathrm{g} \mathrm{ha}^{-1}$ de i.a. em avaliações realizadas até os 21 DAT. No entanto, Sikkema et al. (2008) trabalhando com doses de 35 e $70 \mathrm{~g} \mathrm{ha}^{-1}$ de i.a. de saflufenacil encontrou fitotoxicidade leve na cultura da aveia preta e do trigo, mas não ocorreram perdas de produtividade.

Vargas e Roman (2005) também estudaram a seletividade de herbicidas em cereais de inverno, e encontraram resultados que corroboram com este trabalho, onde eles observaram que os herbicidas 2,4-D (806 e $1612 \mathrm{~g} \mathrm{ha}^{-1}$ de i.a.) e bentazona (900 e $1800 \mathrm{~g} \mathrm{ha}^{-1}$ de i.a.) não provocaram fitotoxicidade pronunciada na cultura do azevém em avaliações realizadas até os 40 DAT. Esses mesmos autores também observaram fitotoxicidade na cultura da cevada quando aplicaram 3,6 $\mathrm{g} \mathrm{ha}^{-1}$ de i.a. de metsulfurom-metílico, com percentuais de 8 , 15 e 5\% de fitotoxicidade aos 7, 14 e 25 DAT, respectivamente. Apesar do saflufenacil e do metsulfurom-metílico terem causado fitotoxicidade mais pronunciada e persistente no azevém, todos os herbicidas aplicados mostraram-se seletivos ao mesmo.

$\mathrm{Na}$ avaliação de massa seca da parte aérea (MSPA) pode-se observar que os tratamentos com o herbicida 2,4-D diferiram dos demais tratamentos, apresentando os maiores valores de MSPA de azevém, com 1646,5 e 1590,6 $\mathrm{kg} \mathrm{ha}^{-1}$ para as doses de 806 e $1612 \mathrm{~g} \mathrm{ha}^{-1}$ de i.a., respectivamente. Esses mesmos tratamentos apresentaram os menores valores de MSPA de E. plantagineum, com 123,7 e 82,8 kg ha-1 (806 e $1612 \mathrm{~g} \mathrm{ha}^{-1}$ de i.a.) (Tabela 4). Esses valores estão de acordo com os resultados anteriores de controle e fitotoxicidade, mostrando que ocorreu controle eficiente das plantas de E. plantagineum pelo herbicida 2,4-D e também, que a fitotoxicidade ocorrida nas primeiras avaliações não repercutiram em perdas de produtividade de MSPA. Além disso, o tratamento com 2,4-D + metsulfurom-metílico apresentou resultados semelhantes aos tratamentos anteriores, possuindo MSPA de $1522,5 \mathrm{~kg} \mathrm{ha}^{-1}$, sendo menor que os tratamentos com 2,4-D provavelmente em decorrência da fitotoxicidade apresentada por este tratamento que interferiu no desenvolvimento das plantas de azevém.

Para o herbicida saflufenacil, cabe ressaltar que a MSPA possuiu valores ao redor de $1250 \mathrm{~kg} \mathrm{ha}^{-1}$, para ambas as doses, sendo esses os menores valores encontrados quando comparados aos demais tratamentos, diferindo estatisticamente, provavelmente devido à fitotoxicidade apresentada pelas plantas de azevém e da interferência das plantas de $E$. plantagineum, que apresentaram valores de MSPA ao redor dos $300 \mathrm{~kg} \mathrm{ha}^{-1}$. Pois, a partir dos 21 DAT, essa planta daninha começou a apresentar sintomas de rebrotas, interferindo no desenvolvimento das plantas de azevém.

Os tratamentos com metsulfurom-metílico possuíram valores médios de MSPA de azevém e de E. plantagineum. Para o azevém a MSPA foi de 1287,4 e 1327,7 kg ha-1 (3 e 6 
$\mathrm{g} \mathrm{ha}^{-1}$ de i.a.) e para E. plantagineum foi de 193,8 e 182,3 $\mathrm{kg} \mathrm{ha}^{-1}$ (3 e $6 \mathrm{~g} \mathrm{ha}^{-1}$ de i.a.) (Tabela 4). Esses valores indicam que provavelmente a fitotoxicidade interferiu na MSPA do azevém, juntamente com a interferência das plantas de E. plantagineum, já que os tratamentos apresentaram controle ineficiente dessa planta daninha.

Tabela 4. Massa seca da parte aérea (MSPA) $\left(\mathrm{kg} \mathrm{ha}^{-1}\right)$ de azevém e Echium plantagineum coletadas aos 28 DAT (dias após a aplicação dos tratamentos) em resposta à aplicação de diferentes herbicidas e doses.

\begin{tabular}{|c|c|c|c|c|}
\hline \multirow{2}{*}{$\mathrm{N}^{\circ}$} & \multirow{2}{*}{ Tratamentos } & \multirow{2}{*}{$\begin{array}{c}\text { Dose } \mathrm{g} \mathrm{ha}^{-1} \\
\text { (i.a.) }\end{array}$} & \multicolumn{2}{|r|}{ MSPA } \\
\hline & & & azevém $\left(\mathrm{kg} \mathrm{ha}^{-1}\right.$ & E. plantagineum $\left(\mathrm{kg} \mathrm{ha}^{-1}\right)$ \\
\hline 1 & Testemunha & - & $1203,0 \mathrm{~d}^{*}$ & $563,8 \mathrm{a}$ \\
\hline 2 & Saflufenacil & 28 & $1244,0 \mathrm{~cd}$ & $305,8 \mathrm{~b}$ \\
\hline 3 & Saflufenacil & 56 & $1251,3 \mathrm{~cd}$ & $301,0 \mathrm{bc}$ \\
\hline 4 & $2,4-\mathrm{D}$ & 806 & 1646,5 a & $123,7 \mathrm{~d}$ \\
\hline 5 & $2,4-\mathrm{D}$ & 1612 & $1590,6 \mathrm{ab}$ & $82,8 \mathrm{~d}$ \\
\hline 6 & Metsulfurom-metílico & 3 & $1287,4 \mathrm{~cd}$ & $193,8 \mathrm{bcd}$ \\
\hline 7 & Metsulfurom-metílico & 6 & $1327,7 \mathrm{bcd}$ & $182,3 \mathrm{bcd}$ \\
\hline 8 & Bentazona & 600 & $1391,2 \mathrm{abcd}$ & $323,4 \mathrm{~b}$ \\
\hline 9 & Bentazona & 900 & $1221,9 \mathrm{~d}$ & $309,3 \mathrm{~b}$ \\
\hline \multicolumn{2}{|c|}{10 2,4-D + Metsulfurom-metílico } & $806+3$ & $1522,5 \mathrm{abc}$ & $125,8 \mathrm{~cd}$ \\
\hline & $\mathrm{CV}(\%)^{* *}$ & & 8,47 & 28,72 \\
\hline
\end{tabular}

* Médias seguidas pela mesma letra, na coluna, não diferem significativamente pelo teste de Tukey ao nível de $5 \%$ de probabilidade. ** Coeficiente de variação percentual.

Já os tratamentos com o herbicida bentazona obtiveram MSPA de 1391,2 e 1221,9 kg $\mathrm{ha}^{-1}$ de azevém e 323,4 e 309,3 $\mathrm{kg} \mathrm{ha}^{-1}$ de E. plantagineum para as doses de 600 e $900 \mathrm{~g} \mathrm{ha}^{-1}$ de i.a. (Tabela 4), respectivamente. Esses valores baixos de MSPA de azevém a altos de $E$. plantagineum podem estar relacionados à baixa eficiência desse herbicida no controle das plantas de E. plantagineum, que passaram a interferir no desenvolvimento das plantas de azevém, competindo principalmente por água, luz, nutrientes e espaço.

A testemunha, sem aplicação de herbicida, obteve MSPA de $563,8 \mathrm{~kg} \mathrm{ha}^{-1}$ de $E$. plantagineum e de $1203,0 \mathrm{~kg} \mathrm{ha}^{-1}$ de azevém. Esses valores podem estar atrelados à interferência que as plantas de E. plantagineum provocaram no desenvolvimento do azevém, mostrando redução da MSPA e consequentemente perdas de produtividade da cultura.

Embora esse trabalho tenha mostrado diferença significativa de MSPA entre os tratamentos avaliados, Dalazen et al. (2015) trabalhando com aplicação de 2,4-D (806 e $1612 \mathrm{~g} \mathrm{ha}^{-1}$ de i.a.), metsulfurom-metílico (2,4 e 4,8 $\mathrm{g} \mathrm{ha}^{-1}$ de i.a.) e saflufenacil (35 e $70 \mathrm{~g}$ $\mathrm{ha}^{-1}$ de i.a.) em azevém comum não encontrou diferença significativa de MSPA em relação à testemunha sem aplicação de herbicida. Seus resultados, entretanto, foram obtidos em casa-de-vegetação.

Cultura Agronômica, Ilha Solteira, v.26, n.3, p.297-309, 2017 
Diante disso, fica evidente que E. plantagineum tem capacidade de competir com o azevém causando perdas de massa seca, no entanto, os herbicidas 2,4-D e metsulfurommetílico, aplicados de forma isolada ou conjunta, são alternativas eficientes de controle desta planta daninha. Mas, para o controle ser satisfatório esta planta daninha deve estar em estádios iniciais de desenvolvimento, contribuindo para a rápida ação do herbicida na planta, minimizando a possibilidade de ocorrer o rebrote.

\section{CONCLUSÃO}

O herbicida 2,4-D é eficiente no controle de E. plantagineum. Além disso, apresenta seletividade à cultura apresentando apenas leve fitotoxicidade, não repercutindo em perdas de produtividade de matéria seca.

O tratamento 2,4-D + metsulfurom-metílico é eficiente no controle de E. plantagineum, no entanto, causam fitotoxicidade ao azevém, e consequentemente, redução da massa seca da parte aérea quando comparada aos tratamentos com 2,4-D.

Os tratamentos com saflufenacil proporcionam controle moderado de E. plantagineum, e apresentam fitotoxicidade moderada no azevém, acarretando em diminuição de produtividade da massa seca da parte aérea.

\section{REFERÊNCIAS BIBLIOGRÁFICAS}

BOLDRINI, I. I.; LONGHI-WAGNER, H. M.; BOECHAT, S. C. Morfologia e taxonomia de gramíneas sul-riograndenses. Porto Alegre: Universidade Federal do Rio Grande do Sul, 2008. 96 p.

CONFORTIN, A. C. C.; ROCHA, M. G.; MACHADO, J. M.; ROMAN, J.; QUADROS, F. L. F.; PÖTTER, L. Diferentes massas de forragem sobre as variáveis morfogênicas e estruturais de azevém anual. Ciência Rural, Santa Maria, v. 43, n. 3, p.496-502, 2013.

COSTA, L. O.; RIZZARDI, M. A. Herbicidas alternativos para o controle de Raphanus raphanistrum L. resistente ao herbicida metsulfurom metílico. Revista Brasileira de Herbicidas, Passo Fundo, v. 12, n. 3, p.268-276, 2013.

DALAZEN, G.; KRUSE, N. D.; MACHADO, S. L. O. Herbicidas de uso potencial no controle de buva e sua seletividade sobre aveia e azevém. Revista Ciência Agronômica, Fortaleza, v. 46, n. 4, p.792-799, 2015.

DE CONTO, L.; SGANZERLA, D. C.; PEDROSO, C. E. S.; MONKS, P. L. Relação azevém anual (Lolium multiflorum Lam.) - Ruminante. Arquivos de Zootecnia, Córdoba, v. 60, p. $41-54,2011$.

EMBRAPA. Centro Nacional de Pesquisa de Solos (Rio de Janeiro, RJ). Brasília: Sistema Brasileiro de Classificação dos Solos, 2006. 412 p. 
FARINELLI, R.; PENARIOL, F.G.; LEMOS, L.B. Eficiência do herbicida 2,4-D no controle de Raphanus raphanistrum L., em pós-emergência na cultura de milheto. Revista Brasileira de Milho e Sorgo, v. 4, n. 1, p.104-111. 2005.

FARINATTI, L. H. E.; ROCHA, M. G.; POLI, C. H. E. C.; PIRES, C. C.; PÖTTER, L.; SILVA, J. H. S. Desempenho de ovinos recebendo suplementos ou mantidos exclusivamente em pastagem de azevém (Lolium multiflorum Lam.). Revista Brasileira de Zootecnia, Viçosa, v. 35, n. 2, p.527-534, 2006.

FERRAZZA, J. M.; SOARES, A. B.; MARTIN, T. N.; ASSMANN, A. L.; NICOLA, V. Produção de forrageiras anuais de inverno em diferentes épocas de semeadura. Revista Ciência Agronômica, Fortaleza, v. 44, n. 2, p.379-389, 2013.

FRANS, R. E. Experimental design and techniques for me measuring and analyzing plant responses to weed control practices. In: CAMPER, N. D. (Ed). Research methods in weed science: Southern Weed Science Society. Puerto Rico: Champaign, 1986. Cap 1, p. 28-41.

GROSSMANN, K. R.; HUTZLER, J.; KWIAKOWSKI, J.; BROMMER, C. L. Saflufenacil $\left(\right.$ Kixor $\left.^{\mathrm{TM}}\right)$ : biokinetic properties and mechanism of selectivity of a new protoporphyrinogen IX oxidase inhibiting herbicide. Weed Science, Cambridge, v. 59, n. 3, p.290-298, 2011.

INOUE, M. H.; DUARTE, J. C. B.; MENDES, K. F.; SZTOLTZ, J.; BEM, R.; PEREIRA, R. L. Eficácia de herbicidas aplicados em plantas adultas de Crotalaria spectabilis e Crotalaria ochroleuca. Revista Brasileira de Herbicidas, Passo Fundo, v. 11, n. 2, p.14858, 2012.

MONQUERO, P. A.; CHRISTOFFOLETI, P. J. Manejo de população de plantas daninhas resistentes aos herbicidas inibidores da acetolactato sintase. Planta Daninha, Viçosa, v. 19, n. 1, p.67-74, 2001.

MOREIRA, H. J. da C.; BRAGANÇA, H. A. B. N. Manual de identificação de plantas infestantes: Cultivos de verão. Campinas: FMC, 2010. 326 p.

MOREno, J. A. Clima do Rio Grande do Sul. Porto Alegre: Secretaria da Agricultura, 1961. $41 \mathrm{p}$.

PICCININI, F.; REIMCHE, G.; CASAGRANDE, G. S.; CORADINI, C.; MACHADO, S. L. O. Aplicações isoladas ou associadas de glifosato e saflufenacil para o controle de Ipomoea triloba e Ipomoea purpúrea. Enciclopédia Biosfera, Goiânia, v. 8, n. 15, p.150, 2012.

ROMAN, J.; ROCHA, M. G.; PIRES, C. C.; ELEJALDE, D. A. G.; KLOSS, M. G.; NETO, R. A. O. Comportamento ingestivo e desempenho de ovinos em pastagem de azevém anual (Lolium multiflorum Lam.) com diferentes massas de forragem. Revista Brasileira de Zootecnia, Viçosa, v. 36, n. 4, p.780-788, 2007. 
SAINT-HILAIRE, A. Plantas usuais dos brasileiros. BRANDÃO, M. G. L.; PIGNAL, M. (Orgs.). Tradução: MOURÃO, C. P. B.; SANTIAGO, C. F. Belo Horizonte: IDM Composição e Arte Ltda, 2009. 392 p.

SANTOS, J. C. A.; RIET-CORREA, F.; SIMÕES, S. V. D.; BARROS, C. S. L. Patogênese, sinais clínicos e patologia das doenças causadas por plantas hepatotóxicas em ruminantes e equiinos no Brasil. Pesquisa Veterinária Brasileira, Rio de Janeiro, v. 28, n. 1, p.1-14, 2008.

SANTOS, M. V.; FERREIRA, E. A.; FONSECA, D. M.; FERREIRA, L. R.; SANTOS, L. D. T.; SILVA, D. V. Levantamento fitossociológico e produção de forragem em pasto de capim-gordura. Revista Ceres, Viçosa, v. 62, n. 6, p.561-567, 2015.

SIKKEMA, P. H.; SHROPSHIRE, C.; SOLTANI, N. Tolerance of spring barley (Hordeum vulgare L.), oats (Avena sativa L.) and wheat (Triticum aestivum L.) to saflufenacil. Crop protection, Florida, v. 27, n. 3, p.1495-1497, 2008.

TAKANO, H. K.; JUNIOR, R. S. O.; CONSTANTIN, J.; BIFFE, D. F.; FRANCHINI, L. H. M.; BRAZ, G. B. P.; RIOS, F. A.; GHENO, E. A.; GEMELLI, A. Efeito da adição do 2,4-D ao glyphosate para o controle de espécies de plantas daninhas de difícil controle. Revista Brasileira de Herbicidas, Passo Fundo, v. 12, n. 1, p.1-13, 2013.

VARGAS, L.; MORAES, R. M. A.; BERTO, C. M. Herança da resistência do azevém (Lolium multiflorum) ao glyphosate. Planta Daninha, Viçosa, v. 25, n. 3, p.567-571, 2007.

VARGAS, L.; ROMAN, E. S. Seletividade e eficiência de herbicidas em cereais de inverno. Revista Brasileira de Herbicidas, Passo Fundo, v. 4, n. 3, p.1-10, 2005.

VITORINO, H. S.; MARTINS, D.; COSTA, S. I.; MARQUES, R. P.; SOUZA, G. S. F.; CAMPOS, C. F. Eficiência de herbicidas no controle de plantas daninhas latifoliadas em mamona. Arquivos do Instituto Biológico, São Paulo, v. 79, n. 1, p.127-131, 2012.

WESTON, L. A.; WESTON, P. A.; MCCULLY, M. Production of bioactive napthoquinones by roots of paterson's curse (Echium plantagineum) - Implications for invasion success? Weed Science Research, Cambridge, v. 18, edição especial, p.677-686, 2012. 